\title{
Septic shock due to Yersinia pseudotuberculosis infection in an adult immunocompetent patient: a case report and literature review
}

\author{
Takehiro Hashimoto ${ }^{1}$, Ryuichi Takenaka ${ }^{1,2}$, Haruka Fukuda ${ }^{3}$, Kazuhiko Hashinaga', Shin-ichi Nureki ${ }^{1 *}$, \\ Hideki Hayashidani ${ }^{4}$, Teruo Sakamoto ${ }^{3}$ and Osamu Shigemitsu ${ }^{2}$
}

\begin{abstract}
Background: Yersinia pseudotuberculosis infection can occur in an immunocompromised host. Although rare, bacteremia due to Y. pseudotuberculosis may also occur in immunocompetent hosts. The prognosis and therapeutic strategy, especially for immunocompetent patients with $Y$. pseudotuberculosis bacteremia, however, remains unknown.

Case presentation: A 38-year-old Japanese man with a mood disorder presented to our hospital with fever and diarrhea. Chest computed tomography revealed consolidation in the right upper lobe with air bronchograms. He was diagnosed with pneumonia, and treatment with intravenous ceftriaxone and azithromycin was initiated. The ceftriaxone was replaced with doripenem and the azithromycin was discontinued following the detection of Gramnegative rod bacteria in 2 sets of blood culture tests. The isolated Gram-negative rod bacteria were confirmed to be Y. pseudotuberculosis. Thereafter, he developed septic shock. Doripenem was switched to cefmetazole, which was continued for 14 days. He recovered without relapse.
\end{abstract}

Conclusions: We herein report a case of septic shock due to Y. pseudotuberculosis infection in an adult immunocompetent patient. The appropriate microorganism tests and antibiotic therapy are necessary to treat patients with Y. pseudotuberculosis bacteremia.

Keywords: Azithromycin, Bacteremia, Cefmetazole, Ceftriaxone, Doripenem, Septic shock, Yersinia pseudotuberculosis

\section{Background}

Yersinia pseudotuberculosis is a Gram-negative rod bacterium belonging to the family Enterobacteriaceae. $Y$. pseudotuberculosis is commonly found in contaminated food and water [1]. Transmission to humans is uncommon and occurs through the ingestion of contaminated food, water or milk, or direct contact with an infected

\footnotetext{
*Correspondence: nureki@oita-u.ac.jp

'Department of Respiratory Medicine and Infectious Diseases, Oita University Faculty of Medicine, 1-1 Idaigaoka, Hasama-machi, Yufu, Oita 879-5593, Japan Full list of author information is available at the end of the article
}

animal, such as rodents, rabbits, deer, farm animals, and birds $[1,2]$. Y. pseudotuberculosis infections typically manifest gastroenteritis [2]. A self-limiting acute infection is common. Severe infections and chronic conditions can also occur, however, particularly in immunocompromised patients. Although rare, $Y$. pseudotuberculosis infection may progress to bacteremia in adult immunocompetent patients. The definition of septic shock was changed to be more strict in 2016 [3]. In the literature, there are no case reports fulfilling the new definition of septic shock in adult immunocompetent patients with Y. pseudotuberculosis

(c) The Author(s). 2021 Open Access This article is licensed under a Creative Commons Attribution 4.0 International License, which permits use, sharing, adaptation, distribution and reproduction in any medium or format, as long as you give appropriate credit to the original author(s) and the source, provide a link to the Creative Commons licence, and indicate if changes were made. The images or other third party material in this article are included in the article's Creative Commons licence, unless indicated otherwise in a credit line to the material. If material is not included in the article's Creative Commons licence and your intended use is not permitted by statutory regulation or exceeds the permitted use, you will need to obtain permission directly from the copyright holder. To view a copy of this licence, visit http://creativecommons.org/licenses/by/4.0/ The Creative Commons Public Domain Dedication waiver (http://creativecommons.org/publicdomain/zero/1.0/) applies to the data made available in this article, unless otherwise stated in a credit line to the data. 
infection. We herein report the first case of septic shock due to $Y$. pseudotuberculosis infection in an adult immunocompetent patient. The patient was successfully treated with the appropriate antibiotics.

\section{Case presentation}

A 38-year-old Japanese man with a mood disorder was admitted to the emergency department of Oita University Hospital (Oita, Japan) for complaints of fever and diarrhea. Ten days prior to presentation at the emergency department, he experienced appetite loss and vomiting a few days after attending a riverside barbecue and eating half-roasted foods. He was receiving oral ethyl loflazepate and paroxetine hydrochloride hydrate therapy for mood disorder and alcoholism. The patient had a fever and watery diarrhea 3 days prior to admission. His body temperature was $38.8^{\circ} \mathrm{C}$, blood pressure $111 / 70 \mathrm{mmHg}$, pulse 112 beats/min, and he had an $\mathrm{SpO}_{2}$ of $100 \%$ at $3 \mathrm{~L} /$ min with a nasal mask on admission. Although he was awake without any stimuli, he was unable to recall his name or date of birth. No murmur was detected on heart examination. No crackles were auscultated in either lung field. Laboratory tests revealed an elevated white blood cell count $(11,840 / \mu \mathrm{L})$, hypoalbuminemia $(2.6 \mathrm{~g} / \mathrm{dL})$, decreased serum iron level $(22 \mu / \mathrm{dL})$, decreased serum unsaturated iron-binding capacity level $(162 \mu \mathrm{g} / \mathrm{dL})$, decreased transferrin saturation (TSAT) level (12\%), elevated C-reactive protein level $(9.58 \mathrm{mg} / \mathrm{dL})$, and an elevated procalcitonin $(18.8 \mathrm{ng} / \mathrm{mL})$. A chest X-ray showed an infiltrative shadow in the right upper lung field. Whole body computed tomography showed no remarkable finding except for consolidation with air bronchograms in the right upper lung lobe. The patient was diagnosed with community-acquired pneumonia, and treatment with intravenous ceftriaxone (2 g every $24 \mathrm{~h}$ ) and azithromycin $(500 \mathrm{mg}$ every $24 \mathrm{~h}$ ) was initiated. On day 3, 2 sets of blood culture tests revealed the presence of Gram -negative rod bacteria (Fig. 1-a); therefore, the ceftriaxone was replaced with doripenem (500 mg every $8 \mathrm{~h}$ ) and the azithromycin was discontinued. On day 4, the Gram-negative rod bacteria were identified as $Y$. pseudotuberculosis, using the VITEK 2 system (bioMérieux, Marcy l'Etoile, France) with 99\% probability. Furthermore, matrix-assisted laser-desorption/ionization time-of-flight mass spectrometry (Bruker Daltonics, Billerica, MA, USA) was used to identify the bacteria as $Y$. pseudotuberculosis with highly probable species identification (score value, 2.32). On day 5, the patient's blood pressure decreased to $80 / 50 \mathrm{mmHg}$ (mean arterial pressure $60 \mathrm{mmHg}$ ). Blood gas analysis revealed an elevated lactate level $(4.1 \mathrm{mmol} / \mathrm{L})$. The hypotension persisted despite adequate volume resuscitation, and norepinephrine as a vasopressor was started. The patient was diagnosed with septic shock due to $Y$. pseudotuberculosis infection. On the basis of susceptibility
(A)

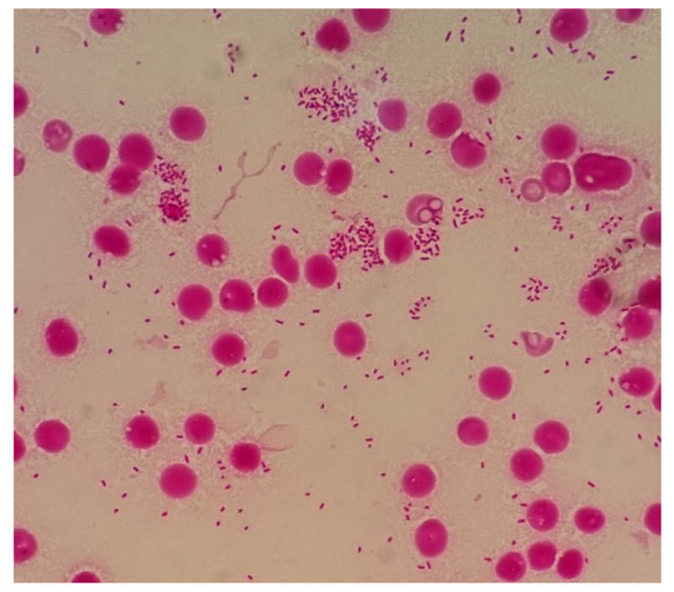

(B)

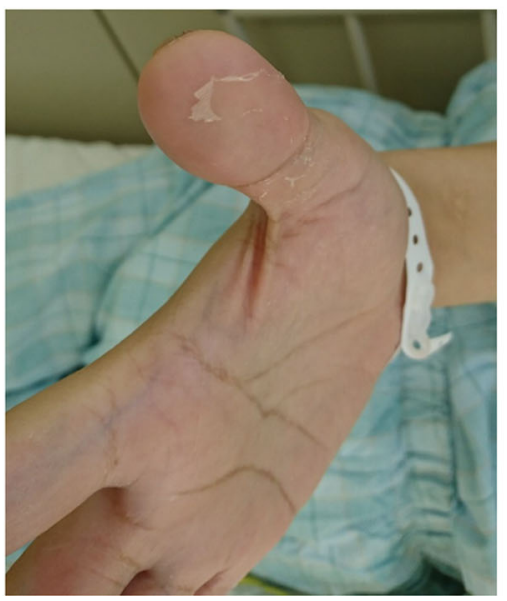

Fig. 1 a Blood culture test detected Gram -negative rod bacteria (Gram stain, magnification $1000 \times$ ) on day 3. b Desquamation of the fingers was observed on day 7

testing of $Y$. pseudotuberculosis using a dry plate (Eiken, Tokyo, Japan) with a conventional microdilution method and analysis by an image analyzer (Koden IA40MIC-i, Koden, Tokyo, Japan), the strain was found to be sensitive to ampicillin, ampicillin/sulbactam, cefotiam, ceftazidime, cefmetazole, meropenem, gentamicin, and levofloxacin. A sputum culture test was negative. A stool culture test detected Escherichia coli and Streptococcus spp, but no $Y$. pseudotuberculosis. On day 7, his fever was reduced and the norepinephrine was discontinued. Desquamation of the fingers was observed (Fig. 1-b). Two sets of blood culture tests showed negative results. On day 9 , the treatment regimen was changed to cefmetazole ( $2 \mathrm{~g}$ every $8 \mathrm{~h})$ and continued for a total of 14 days. On day 10, a chest X-ray showed complete improvement of the infiltrative shadow in the right upper lung field. On day 17, he was discharged from the hospital. Further characteristics of the Y. pseudotuberculosis strain was performed because of the clinical importance of virulence factors of this strain. Y. pseudotuberculosis isolate was of serotype 6 using serotyping 
scheme based on $\mathrm{O}$-antigen. To evaluate the pathogenicity of this isolate, variants of $Y$. pseudotuberculosis-derived mitogen (YPM) superantigens were investigated in the strain using PCR with the following primers: ypmA [ $\left[5^{\prime}-\right.$ CACTTTTCTCTGGAGTAGCG-3' (forward) and 5'GATGTTTCAGAGCTATTGTT-3' (reverse)] and ypmB [(5' ${ }^{\prime}$-TTTCTGTCATTACTGACATTA-3' (forward) and 5'-CCTCTTTCCATCCATCTCTTA-3' (reverse)] [4]. The PCR test using isolated bacteria showed positivity of ypm A. Combined with the results of serotype 6 and genetic detection of Yersinia pseudotuberculosis-derived mitogen A (YPMa), Y. pseudotuberculosis strain belonged to a genetic group 3 (Far East systemic pathogenicity type) [5]. We finally diagnosed with Far East scarlet-like fever (FESLF) caused by $Y$. pseudotuberculosis complicated septic shock.

\section{Discussion and conclusions}

We report a case of septic shock due to Y. pseudotuberculosis infection in an adult immunocompetent patient. $Y$. pseudotuberculosis infection in humans was first described in 1883 [6]. It is a Gram-negative rod bacterium and can grow in temperatures as low as $4{ }^{\circ} \mathrm{C}$ [2]. The incubation period for intestinal $Y$. pseudotuberculosis infection is approximately 3 to 7 days [7]. Cases of $Y$. pseudotuberculosis infection, including gastroenteritis, pseudoappendicitis, bacteremia, pharyngitis, erythema nodosum, reactive arthritis, and syndromes mimicking Kawasaki disease, have been reported $[2,5,8]$. Risk factors for Y. pseudotuberculosis infection are exposure to contaminated food or water, as well as underlying medical conditions such as hepatic cirrhosis, HIV infection, malignancy, anaplastic anemia, thalassemia, iron overload, and diabetes mellitus $[9,10]$. The mortality of bacteremia due to $Y$. pseudotuberculosis is reported to be as high as 75\% [11]. Nine cases of $Y$. pseudotuberculosis bacteremia in adult immunocompetent patients were reported from 1911 through 1994 [2]. Of the 9 patients, 6 (66\%) died. Four cases reported from 1995 to 2020 and our case of $Y$. pseudotuberculosis bacteremia in an adult immunocompetent patient are summarized in Table 1 [12-15]. All 5 patients survived. Although the case numbers of $Y$. pseudotuberculosis bacteremia in adult immunocompetent patients are limited, the prognosis of recent cases of $Y$. pseudotuberculosis bacteremia in adult immunocompetent patients might be better than that of the previous cases. The better outcome may be related to advances in diagnostic techniques and antibiotic therapy. Further studies are required to establish the prognosis of $Y$. pseudotuberculosis bacteremia in adult immunocompetent patients. Alcohol consumption is known to result in an iron overload, which could be a predisposing factor for systemic $Y$. pseudotuberculosis infection. Although serum ferritin level was not examined in our case, low serum iron and TSAT levels implied the low possibility of iron overload. Thus, we considered the patient would be immunocompetent.

FESLF caused by $Y$. pseudotuberculosis infection is a severe inflammatory disease that occurs sporadically and outbreaks in Russia and Japan [5]. FESLF patients with $Y$. pseudotuberculosis infection can be complicated with desquamation at the distal portion of the extremities in convalescent phase $[16,17]$. Desquamation is seen in 83\% of Y. pseudotuberculosis infection cases in childhood [17]; however, few cases have reported desquamation as a complication of $Y$. pseudotuberculosis infection in adults $[16,18]$. Further studies are required to establish the epidemiology of desquamation in $Y$. pseudotuberculosis infection in adults.

In our case, $Y$. pseudotuberculosis strain was of serotype 6 and harbored superantigen gene ypmA. Y. pseudotuberculosis

Table 1 Cases of Yersinia pseudotuberculosis bacteremia in adult immunocompetent patients. "Septic shock" is defined based on a clinical construct of sepsis with persistent hypotension requiring vasopressors to maintain a mean arterial pressure of $\geq 65 \mathrm{mmHg}$ and having a serum lactate level $>2 \mathrm{mmol} / \mathrm{L}$ despite adequate volume resuscitation

\begin{tabular}{|c|c|c|c|c|c|c|c|}
\hline Author & Age/Sex & Probable portal of entry & $\begin{array}{l}\text { Contaminated food } \\
\text { or water exposure }\end{array}$ & Septic shock & Treatment & Outcome & Reference \\
\hline Ljungberg et al. & $54 / M$ & Unknown & Unknown & No & $\begin{array}{l}\mathrm{PCG} \rightarrow \\
\mathrm{CTRX} \rightarrow \\
\mathrm{CPFX}\end{array}$ & Survived & {$[12]$} \\
\hline Ressler et al. & $68 / F$ & Skin and soft tissue & Unknown & No & Unknown & Survived & {$[13]$} \\
\hline Lai et al. & $33 / M$ & Gastrointestinal tract & Unknown & Unknown & $\begin{array}{l}\mathrm{MFLX} \rightarrow \\
\mathrm{CPFX}\end{array}$ & Survived & [14] \\
\hline Mashiba et al. & $22 / F$ & Unknown & None & No & $\begin{array}{l}\mathrm{CEZ} \rightarrow \\
\mathrm{FOM} \rightarrow \\
\mathrm{IPM} / \mathrm{CS}\end{array}$ & Survived & {$[15]$} \\
\hline Our case & 38/M & Gastrointestinal tract & Undercooked food & No & $\begin{array}{l}\mathrm{CTRX} \rightarrow \\
\mathrm{DRPM} \rightarrow \\
\mathrm{CMZ}\end{array}$ & Survived & \\
\hline
\end{tabular}

Age (years old) and sex (F, female; $M$, male). Abbreviations: CEZ cefazolin, CMZ cefmetazole, CPFX ciprofloxacin, CTRX ceftriaxion, DRPM doripenem, FOM fosfomycin, IPM/CS imipenem/cilastatin, MFLX moxifloxacin, PCG penicillin G 
has been classified into serotypes 1 to 15 [5]. Most European $Y$ pseudotuberculosis isolates are of serotypes 1 to 3, whereas serotypes 4 to 15 are primarily found in Asia [5]. YPMa is a superantigenic toxin produced almost by Far Eastern strains [4] which is involved in the pathogenesis of severe inflammatory disease from patient with FESLF. Thus, the clinical isolate from our patient was compatible with the that from FESLF patients.

The definitions of sepsis and septic shock were changed in 2016 [3]. Septic shock can be defined with persistent hypotension requiring vasopressors to maintain a mean arterial pressure of $\geq 65 \mathrm{mmHg}$ and a serum lactate level $>2 \mathrm{mmol} / \mathrm{L}$ despite adequate volume resuscitation. In the previous diagnostic criteria, septic shock was defined as sepsis with hypotension despite adequate fluid resuscitation [19]. Thus, septic shock according to the new definition is more critical than that based on the previous criteria. Lai et al. reported a case of $Y$. pseudotuberculosis infection in an adult patient who developed to septic shock [14], but their case was reported before 2016 and it is unclear whether their case fulfilled the new septic shock criteria [3]. To our knowledge, according to the new septic shock criteria, our case could be the first case of septic shock due to $Y$. pseudotuberculosis infection in an adult immunocompetent patient.

$Y$. pseudotuberculosis exhibits greater susceptibility to antimicrobials other than macrolides. In a murine model, fluoroquinolone therapy is effective against $Y$. pseudotuberculosis infections whereas beta lactam therapy is associated with lower survival or a poor clinical response $[20,21]$. The best antimicrobial therapy for $Y$. pseudotuberculosis infection, however, is not yet established. Further studies are needed to determine the appropriate treatment for $Y$. pseudotuberculosis infections with bacteremia.

In our case, the illness developed a few days after the patient attended a barbecue. For prevention, it is important to take into account whether or not contaminated food and water were consumed raw and to exclude the possibility of secondary contamination due to undercooked food and unboiled water. Although stool culture did not detect $Y$. pseudotuberculosis in our case, bloodstream infection could originate from intestinal infection. Vulnerability of intestinal tract leading to bacteremia might be caused by mucosal damage due to inflammation of $Y$. pseudotuberculosis infection and intestinal edema came from hypoalbuminemia.

In conclusion, we herein report a case of septic shock due to $Y$. pseudotuberculosis infection in an adult immunocompetent patient. This report will help to raise awareness among clinicians that $Y$. pseudotuberculosis bacteremia should be included in the differential diagnosis when patients exhibit fever and diarrhea after consuming undercooked food.

\section{Abbreviation}

Y. pseudotuberculosis: Yersinia pseudotuberculosis

\section{Acknowledgements}

We would like to thank the emergency ward staff for their excellent team work.

\section{Authors' contributions}

TH and SN drafted the manuscript. TH, RT, HF, KH, SN, TS, and OS contributed to deciding the patient's treatment. $\mathrm{HH}$ analyzed the virulence

factors of clinical isolate. All authors have read and approved the manuscript.

\section{Funding}

None.

\section{Availability of data and materials}

All the data on clinical findings are included in the manuscript.

\section{Ethics approval and consent to participate}

The need for approval was waived off by the Institutional Review Board of our institution. Written informed consent was obtained from the patient on publication of this case report.

\section{Consent for publication}

Written informed consent was obtained from the patient on publication including personal or clinical details along with any identifying images of this case report.

\section{Competing interests}

The authors declare that they have no competing interests.

\section{Author details}

${ }^{1}$ Department of Respiratory Medicine and Infectious Diseases, Oita University Faculty of Medicine, 1-1 Idaigaoka, Hasama-machi, Yufu, Oita 879-5593, Japan. ${ }^{2}$ Department of Emergency Medicine, Oita University Faculty of Medicine, 1-1 Idaigaoka, Hasama-machi, Yufu, Oita 879-5593, Japan.

${ }^{3}$ Advanced Trauma, Emergency, and Critical Care Center, Oita University Hospital, 1-1 Idaigaoka, Hasama-machi, Yufu, Oita 879-5593, Japan. ${ }^{\text {DDivision }}$ of Animal Life Science, Institute of Agriculture, Tokyo University of

Agriculture and Technology, 3-5-8 Saiwai-cho, Fuchu, Tokyo 183-8509, Japan.

Received: 8 July 2020 Accepted: 22 December 2020

Published online: 07 January 2021

\section{References}

1. Galindo CL, Rosenzweig JA, Kirtley ML, Chopra AK. Pathogenesis of $Y$ enterocolitica and $Y$ pseudotuberculosis in Human Yersiniosis. J Pathog. 2011;2011:182051

2. Kaasch AJ, Dinter J, Goeser T, Plum G, Seifert H. Yersinia pseudotuberculosis bloodstream infection and septic arthritis: case report and review of the literature. Infection. 2012:40(2):185-90.

3. Singer M, Deutschman CS, Seymour CW, Shankar-Hari M, Annane D, Bauer M, Bellomo R, Bernard GR, Chiche JD, Coopersmith CM, et al. The third international consensus definitions for sepsis and septic shock (Sepsis-3). JAMA. 2016;315(8):801-10.

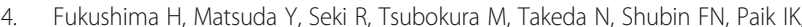
Zheng XB. Geographical heterogeneity between Far East and Western countries in prevalence of the virulence plasmid, the superantigen Yersinia pseudotuberculosis derived mitogen and the high-pathogenicity island among Yersinia pseudotuberculosis strains. J Clin Microbiol. 2001;39(10): $3541-7$

5. Amphlett A. Far East Scarlet-Like Fever: A Review of the Epidemiology, Symptomatology, and Role of Superantigenic Toxin: Yersinia pseudotuberculosis-Derived Mitogen A. Open Forum Infect Dis. 2015;3(1): ofv202.

6. Malassez L, Vignal W. Sur le micro-organisme de la tuberculose zoogloéique. Arch Physiol Norm Pathol. 1884;4:81-104.

7. Smego RA, Frean J, Koornhof HJ. Yersiniosis I: microbiological and clinicoepidemiological aspects of plaque and non-plaque Yersinia infections. Eur J Clin Microbiol Infect Dis. 1999;18(1):1-15 
8. Horinouchi T, Nozu K, Hamahira K, Inaguma Y, Abe J, Nakajima H, Kugo M, lijima K. Yersinia pseudotuberculosis infection in Kawasaki disease and its clinical characteristics. BMC Pediatr. 2015;15:177.

9. Vincent P, Leclercq A, Martin L, Duez JM, Simonet M, Carniel E. Sudden onset of pseudotuberculosis in humans, France, 2004-05. Emerg Infect Dis. 2008;14(7):1119-22.

10. Mischnik A, Dahme T, Bekeredjian R, Zimmermann S. Haemophiliaassociated Yersinia pseudotuberculosis serotype 0:1 septicaemia: the role of iron. J Med Microbiol. 2012;61(Pt 1):157-9.

11. Bennett J, Dolin R, Blaser M. Mandell, Douglas, and Bennett's principles and practice of infectious diseases 8th Edition; 2014.

12. Ljungberg P, Valtonen M, Harjola VP, Kaukoranta-Tolvanen SS, Vaara M. Report of four cases of Yersinia pseudotuberculosis septicemia and a literature review. Eur J Clin Microbiol Infect Dis. 1995;14(9):804-10.

13. Resseler S, Elewaut A, Verhaegen J, ., Colaert J: Yersinia Pseudotuberculosis, a rare cause of septicemia. Clin Microbiol Newsl 2007, 29(10):76-77.

14. Lai CH, Lin JN, Chen YH, Chang LL, Huang WY, Ku HP, Lin HH. The first imported human case of Yersinia pseudotuberculosis serotype $\mathrm{O} 1$ septicemia presents with acute appendicitis-like syndrome in Taiwan. J Formos Med Assoc. 2014;113(9):656-9.

15. Mashiba K, Kojima T, Ootsuka T, Kusaba T. Yersinia psuedotuberculosis septicemia in a healthy young woman. Kansenshogaku Zasshi. 2008; 82(2):86-9.

16. Ocho K, Iwamuro M, Hasegawa K, Hagiya H, Rai K, Yumoto T, Otsuka F. Far East scarlet-like fever masquerading as adult-onset Kawasaki disease. Intern Med. 2018:57(3):437-40.

17. Sato K. Clinical findings and epidemiology of Yersinia pseudotuberculosis infection, especially concerned with Izumi fever. Kansenshogaku Zasshi. 1987;61 (7):746-62 [Article in Japanese].

18. Kawasaki A, Tsuji K, Doyama H. Multiple small intestine ulcers with desquamation of the fingers. Case Rep Gastroenterol. 2017;11(2):452-61.

19. American College of Chest Physicians/Society of Critical Care Medicine Consensus Conference: definitions for sepsis and organ failure and guidelines for the use of innovative therapies in sepsis. Crit Care Med. 1992; 20(6):864-7.

20. Hoogkamp-Korstanje JA. Antibiotics in Yersinia enterocolitica infections. J Antimicrob Chemother. 1987;20(1):123-31.

21. Lemaitre BC, Mazigh DA, Scavizzi MR. Failure of beta-lactam antibiotics and marked efficacy of fluoroquinolones in treatment of murine Yersinia pseudotuberculosis infection. Antimicrob Agents Chemother. 1991;35(9): 1785-90.

\section{Publisher's Note}

Springer Nature remains neutral with regard to jurisdictional claims in published maps and institutional affiliations.

Ready to submit your research? Choose BMC and benefit from:

- fast, convenient online submission

- thorough peer review by experienced researchers in your field

- rapid publication on acceptance

- support for research data, including large and complex data types

- gold Open Access which fosters wider collaboration and increased citations

- maximum visibility for your research: over $100 \mathrm{M}$ website views per year

At $\mathrm{BMC}$, research is always in progress.

Learn more biomedcentral.com/submissions 